\title{
Potentiometric Titration Behavior of Poly(crotonic acid)
}

\author{
Yoshio Muroga and Mitsuru NagasawA \\ Department of Synthetic Chemistry, Faculty of Engineering, Nagoya University, \\ Furo-cho, Chikusa-ku, Nagoya, 464, Japan
}

(Received July 2, 1985)

\begin{abstract}
The potentiometric titration curve of poly(crotonic acid) was compared with that of poly(methacrylic acid). Poly(crotonic acid) is a far weaker acid than poly(methacrylic acid), despite the fact that the conformation of poly(crotonic acid) is a random coil, similar to that of poly(methacrylic acid). This difference is ascribed to their difference in the relative position of the methyl group to the carboxyl group. The methyl group in poly(crotonic acid) intervenes between two adjacent carboxyl groups, leading to the decrease in the local dielectric constant effective for ionization of carboxyl groups.
\end{abstract}

KEY WORDS Potentiometric Titration / Poly(crotonic acid) / Poly(methacrylic acid) / Local Dielectric Constant / Local Conformation / Hydrophobic Force / Dissociation Constant / Conformational Transition /

The molecular structure of poly(crotonate)s is different from that of poly(methacrylate)s only in the position of the methyl group. However, such a difference in the position of the methyl group can give great effects on properties of the polymer chains. One example may be its effect on the stiffness of polymer chains. If the ester group is a bulky one such as tert-butyl group, the poly(crotonate) chain is much stiffer than that of poty(methacrylate) chain because of the steric hindrance between the bulky ester group and the vicinal $\beta$-methyl group. $^{1}$

Besides, the rotational motion around main chains does not change the relative position of the geminal $\alpha$-methyl group to the carboxylate group in poly(methacrylate)s, whereas the motion can change the relative positions of the vicinal $\beta$-methyl group to the carboxylate group in poly(crotonate)s. Therefore, the $\beta$ methyl group in poly(crotonic acid) intervenes between adjacent carboxylate groups and affect the electrostatic interaction between charged groups. The aim of this paper is to show the importance of local conformation and configuration in the dissociation behavior of polyacids by comparing the dissociation behavior of poly(crotonic acid) with that of poly(methacrylic acid).

\section{EXPERIMENTAL}

\section{Samples}

Isotactic and atactic poly(crotonic acid)s, PCA's, were prepared by hydrolysis of the corresponding poly(tert-butyl crotonate), PTBC, in concd sulfuric acid. ${ }^{1}$ PTBC was dissolved either directly in concd $\mathrm{H}_{2} \mathrm{SO}_{4}$ or in $\mathrm{CHCl}_{3}$. In the latter case, concd $\mathrm{H}_{2} \mathrm{SO}_{4}$ was then added. The solutions were heated in a water bath for several hours. Vigorous bubbling nitrogen gas was supplied to prevent oxidation of the sample and to remove the gaseous product. Solutions were then diluted with water and dialized against water. Aqueous polymer solutions thus obtained were neutralized with $\mathrm{NaOH}$, and then purified with ion exchange resins.

The degree of hydrolysis (D.H.) of PCA was calculated from its proton NMR spectrum, 

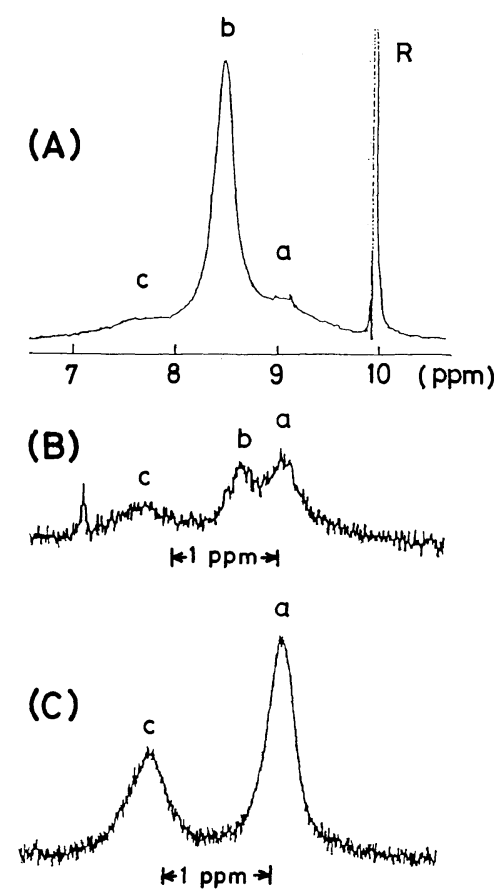

Figure 1. (A) ${ }^{1} \mathrm{H}$ NMR spectrum of poly(tert-butyl crotonate) (sample O-202) in about $5 \mathrm{w} / \mathrm{v} \% \mathrm{CDCl}_{3}$ solution at room temperature. Letters represent all protons in this polymer and tetramethylsilane as a reference: $\mathrm{R}$, TMS; a, $\beta-\mathrm{CH}_{3} ; \mathrm{b}, \mathrm{CH}_{3}$ in tert-butyl group; $\mathrm{c}, \alpha-\mathrm{CH}$ and $\beta$ - $\mathrm{CH}$.

(B) ${ }^{1} \mathrm{H}$ NMR spectrum of sodium crotonate-tert-butyl crotonate copolymer (sample $\mathrm{O}^{\prime}-202$ ) in about $5 \mathrm{w} / \mathrm{v} \%$ $\mathrm{D}_{2} \mathrm{O}$ solution at room temperature. Letters represent all protons in this polymer: a, $\beta-\mathrm{CH}_{3} ; \mathrm{b}, \mathrm{CH}_{3}$ in tert-butyl group; $\mathrm{c}, \alpha-\mathrm{CH}$ and $\beta-\mathrm{CH}$.

(C) ${ }^{1} \mathrm{H}$ NMR spectrum of poly(sodium crotonate) (sample C-105) in about $5 \mathrm{w} / \mathrm{v} \% \quad \mathrm{D}_{2} \mathrm{O}$ solution at room temperature. Letters represent all protons in this polymer: a, $\beta-\mathrm{CH}_{3} ; \mathrm{c}, \alpha-\mathrm{CH}$ and $\beta-\mathrm{CH}$.

recorded by a JNM-PMX 60 spectrometer (Japan Electron Optics Laboratory, Tokyo, Japan). Their typical examples are shown in Figures 1A, 1B, and 1C. Figure 1A shows the NMR spectrum of parent atactic PTBC (sample O-202), Figure 1B that of the partially hydrolyzed sample of O-202 (sample $\mathrm{O}^{\prime}-202$ ) and Figure $1 \mathrm{C}$ that of the completely hydrolyzed sample (sample C-105). Each hump of O-202, a, b, and c in Figure 1A, can be easily assigned to the $\beta$-methyl group, methyl group in tert-butyl group and the sum of the $\alpha$ - and $\beta$-methine groups, respectively, based on their chemical shifts and ratio of their areas. In the partially hydrolyzed sample, $\mathrm{O}^{\prime}-202$, there still remains a hump of methyl group in tert-butyl group (hump b in Figure 1B), but this hump disappears in the completely hydrolyzed sample, C-105.

D. H. may be give by

$$
\text { D.H. }=1-\frac{S_{2}-\frac{3}{2} \times S_{1}}{\frac{S_{1}}{2} \times 9}
$$

where $S_{1}$ and $S_{2}$ represent the areas under the humps assigned to the sum of the $\alpha$ - and $\beta$ methine groups (hump c) and the sum of methyl group in tert-butyl group (hump b) and $\beta$-methyl group (hump a), respectively. D. $\mathrm{H}$. thus determined was almost $100 \%$ for all atactic (samples C-105 and C-108) and isotactic (sample C-ISO) samples, while $71 \%$ for atactic $\mathrm{O}^{\prime}-202$.

The number-averaged molecular weight, $M_{n}$, of poly(sodium crotonate), $\mathrm{PNaC}$, was measured in $0.1 \mathrm{~N}$ aqueous solution of $\mathrm{NaCl}$ at $25^{\circ} \mathrm{C}$ with a Hewlett Packard high-speed membrane osmometer type 502. $M_{n}$ of atactic $\mathrm{PNaC}\left(\mathrm{C}-105,-108, \mathrm{O}^{\prime}-202\right)$ and isotactic $\mathrm{PNaC}$ (C-ISO) were $1.9 \times 10^{4}, 2.2 \times 10^{4}$, $10.0 \times 10^{4}$ and $2.3 \times 10^{4}$, respectively.

The tacticity of PCA was assumed to be identical with that of the corresponding parent PTBC, and the latter was determined by carbon-13 NMR spectra, run on a $25.2 \mathrm{MHz}$ Varian XL-100 spectrometer. The tacticities of PCA's were as follows: $(\mathrm{rRrR})=35 \pm 5$, $(\mathrm{mMrR})=30 \pm 5$, and $(\mathrm{mMmM})=35 \pm 5 \%$ for isotactic PCA (C-ISO), while $(\mathrm{rR})+(\mathrm{mR})=$ $30 \pm 5, \quad(\mathrm{mM})=70 \pm 5, \quad(\mathrm{Rr})+(\mathrm{Mr})=30 \pm 5$, and $(\mathrm{Mm})=70 \pm 5 \%$ for atactic PCA (C-105 and $\mathrm{C}-108)$, if expressed in the same notation as used in our previous paper. ${ }^{2)}$ The tacticity of atactic PTBC O-202 was not determined.

The sodium salts of all samples were con- 
verted to acid forms by passing them through a mixed-bed ion-exchange-resin column of Amberlite IR 120 and 400. Aqueous solutions of PCA obtained were colorless and transparent.

Potentiometric Titration: Measurements of $\mathrm{pH}$ were carried out at $25 \pm 0.1{ }^{\circ} \mathrm{C}$ with a Beckman Research $\mathrm{pH}$ meter in an atmosphere of argon. The concentrations of $\mathrm{NaCl}$ added, $C_{\mathrm{s}}$, were 0.01 and $0.1 \mathrm{~N}$. Concentrations of the sample solutions, $C_{\mathrm{p}}$ 's, were adjusted in the range from 0.003 to $0.010 \mathrm{~N}$.

\section{RESULTS AND DISCUSSION}

The $\mathrm{pH}$ of polyelectrolyte solutions is given by $^{3-7}$

$$
\mathrm{pH}=\mathrm{p} K_{0}-\log \left(\frac{1-\alpha}{\alpha}\right)+\frac{0.434}{R T} \cdot \frac{\partial G_{\mathrm{el}}}{\partial \alpha}
$$

where $K_{0}$ is the intrinsic dissociation constant of a carboxyl group of the polyacid, $\alpha$ is the ionization degree of carboxyl groups, which is the ratio of the amount of $\mathrm{NaOH}$ added to the solution to that needed for complete neutralization, and $\partial G_{\mathrm{el}} / \partial \alpha$ is the change of the Gibbs free energy due to dissociation of the carboxyl group. If we define the apparent dissociation constant of the polyacid $K_{\mathrm{a}}$ by

$$
\mathrm{p} K_{\mathrm{a}}=\mathrm{pH}+\log \left(\frac{1-\alpha}{\alpha}\right)
$$

Eq 2 becomes

$$
\mathrm{p} K_{\mathrm{a}}=\mathrm{p} K_{0}+\frac{0.434}{R T} \cdot \frac{\partial G_{\mathrm{el}}}{\partial \alpha}
$$

The potentiometric titration curves of isotactic and atactic PCA are shown in the form of $\mathrm{p} K_{\mathrm{a}}$ $v s . \alpha$ in Figures $2 \mathrm{~A}\left(C_{\mathrm{s}}=0.1 N\right)$ and $2 \mathrm{~B}$ $\left(C_{\mathrm{s}}=0.01 \mathrm{~N}\right)$, together with those of atactic poly(methacrylic acid), PMAA. ${ }^{8}$ Their polymer concentrations were so low that the polymer concentration dependence of the potentiometric titration curves may be assumed negligible. The $\mathrm{p} K_{0}$ 's can be obtained by extrapolating the titration curves to $\alpha=0$. They are 4.7 to 4.8 for both PCA and PMAA in $0.1 N \mathrm{NaCl}$ solution. There is no difference in the intrinsic acidity of a carboxyl group in either polyacid. Moreover, these $\mathrm{p} K_{0}$ values seem to be reasonable judging from the $\mathrm{p} K$ 's of methacrylic acid and crotonic acid, $4.66^{9}$ (at $18^{\circ} \mathrm{C}$ ) and $4.698^{\circ}$ (at $25^{\circ} \mathrm{C}$ ), respectively.

However, the overall titration behavior of PCA is clearly distinct from that of PMAA in two respects. PCA is a far weaker acid than PMAA at high values of $\alpha$, and a hump at low $\alpha$, which is always observed for PMAA, ${ }^{8}$ cannot be observed in the titration curve of PCA. As reported by Leyte and Mandel, ${ }^{10}$ the hump observed for PMAA appears as a result of the conformational transition from a compact gloular structure, formed by hydrophobic bonding of methyl groups, to a random coil. The reason why this feature is not observed in PCA is not clear.

In Figure $2 \mathrm{~A}$ the titration curve of the random copolymer of crotonic acid and tertbutyl crotonate, $\mathrm{O}^{\prime}-202$, at $C_{\mathrm{s}}=0.1 \mathrm{~N}$, is also shown for comparison. In the figure, $\alpha$ of $\mathrm{O}^{\prime}$ 202 on the abscissa is expressed as the mole ratio of ionized carboxyl groups to the total carboxyl and tert-butyl carboxylate groups. In the titration curve of $\mathrm{O}^{\prime}-202$, a hump similar to that for PMAA at low $\alpha$ is observed. Considering that a part of hydrophobic tertbutyl groups still remains in $\mathrm{O}^{\prime}-202$, it may be concluded that a compact globular structure may be formed in $\mathrm{O}^{\prime}-202$ due to the hydrophobic forces of tert-butyl and $\beta$-methyl groups and a transition to a more extended random coil takes place just as in PMAA as $\alpha$ increases beyond 0.1 . From these observations, we conclude that the conformation of PCA may be represented by random coils similar to the conformation of PMAA over the entire range of $\alpha$. This conclusion is supported by the experimental result, ${ }^{1}$ obtained by small angle $\mathrm{X}$-ray scattering, that the persistence length of $\mathrm{PNaC}$ ( $c a .11 \AA$ ) is comparable to those of randomly coiled vinyl polymers over a wide range of $\alpha$. 


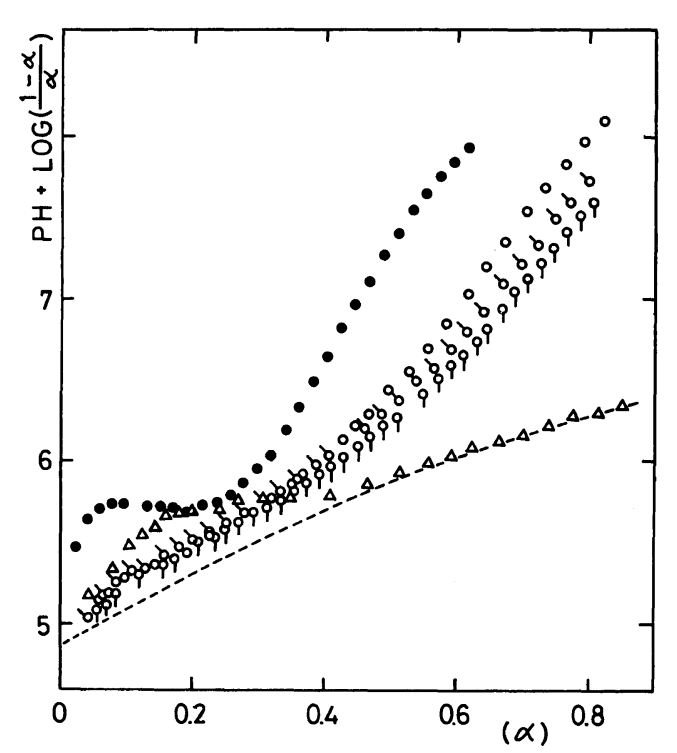

Figure 2A. Potentiometric titration curves of poly(crotonic acid), PCA, crotonic acid-tert-butyl crotonate copolymer, $\mathrm{O}^{\prime}-202$, and poly(methacrylic acid), PMAA, at $\mathrm{C}_{\mathrm{s}}=0.1 N$. (a) Open circles show the data of PCA: $O, \quad$ C-ISO $\quad\left(C_{\mathrm{p}}=0.004 N\right) ; \quad \bigcirc, \quad \mathrm{C}-108$ $\left(C_{\mathrm{p}}=0.008 N\right)$;, $\mathrm{C}-105\left(C_{\mathrm{p}}=0.009 N\right)$. (b) Filled circles shows the data of $\mathrm{O}^{\prime}-202$ at $C_{\mathrm{p}}=0.003 N$. (c) Open triangle show the data of PMAA, cited from ref 8 . The broken line is the theoretical curve, cited from the same reference.

Nevertheless, the observed titration curve for PCA deviates upward from that of PMAA. This difference can be caused by the difference in the position of methyl group in the two samples. That is, the $\beta$-methyl group can intervene in the electrostatic interaction between charged groups, decreasing the dielectric constant of the medium between ionizable groups in $\mathrm{PNaC}$, whereas such an intervening effect of methyl group cannot occur in poly(sodium methacrylate), PNaM. Moreover, it is understandable that the potentiometric titration curve of PCA does not agree with the calculated one ${ }^{8}$ (dotted lines in Figures $2 \mathrm{~A}$ and 2B), obtained by assuming that the dielectric constant is equal to that of pure water. The fact that $\mathrm{O}^{\prime}-202$ is a weaker acid than PCA

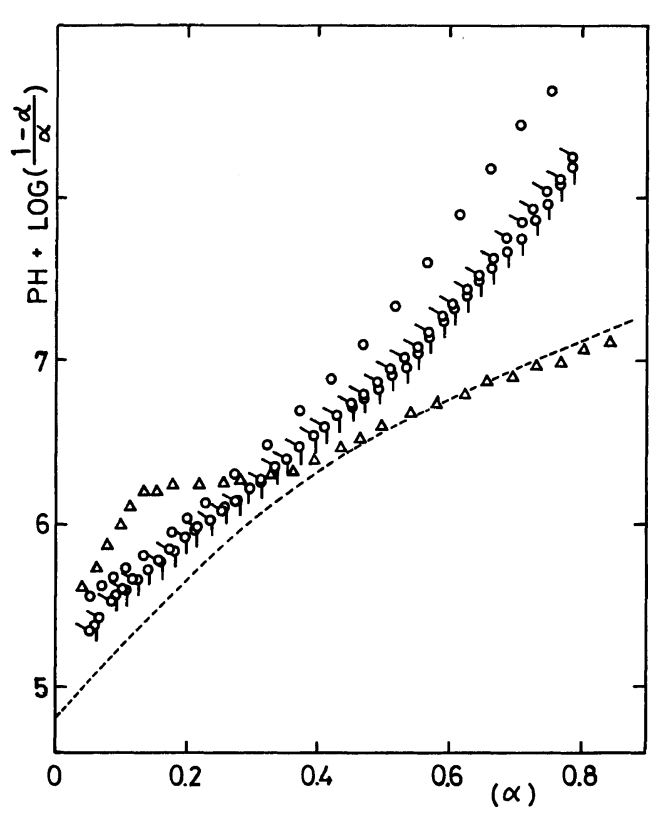

Figure 2B. Potentiometric titration curves of PCA and PMAA at $C_{\mathrm{s}}=0.01 N$. (a) Open circles show the data of PCA: O, C-ISO $\left(C_{\mathrm{p}}=0.002 N\right) ; \bigcirc, \mathrm{C}-105\left(C_{\mathrm{p}}=0.010 N\right)$; O, C-105 $\left(C_{\mathrm{p}}=0.003 N\right)$. (b) Open triangle show the data of PMAA, cited from ref 8 . The broken line is the theoretical curve, cited from the same reference.

in the region of $a>0.3$ may also be due to the additional intervening effect of ester. groups.

It - was already pointed out that the dissociation behavior of polyacids is very sensitive to a change in the local conformation of the polyelectrolyte. ${ }^{11,12}$ In this paper, it is pointed out that a change in the position of methyl group gives a great effect on the dissociation behavior of polyacids through the local dielectric constant around acid groups. ${ }^{13}$ At present, however, the effect of local dielectric constant cannot properly be taken into account in the analysis of dissociation behavior of polyacids.

In a series of studies on the dissociation behavior of $-\mathrm{COOH}$ in various polyelectrolytes, Sakaguchi et al. ${ }^{14,15}$ reported that poly(crotonic acid), prepared by radical polymerization of crotonic acid monomer in bulk, is a much stronger acid than ordinary 
poly(acrylic acid) in disagreement with the conclusion in the present work. The reason for the disagreement may be clarified when the molecular structures and molecular weights of two samples used are carefully compared. It is generally believed ${ }^{16}$ that crotonic acid cannot be radically homopolymerized, and the acidity of polyacids markedly varies with molecular weight if the molecular weight of the polyacid is not above a certain value.

Acknowledgments. The authors thank Mr. Koichiro Hori and Mr. Satoshi Yukioka of Nagoya University for their help in making the potentiometric titration measurements.

\section{REFERENCES}

1. Y. Muroga, I. Sakuragi, I. Noda, and M. Nagasawa, Macromolecules, 17, 1844 (1984).

2. Y. Muroga, I. Noda, and M. Nagasawa, Macromolecules, 13, 1081 (1980).

3. J. Th. G. Overbeek, Bull. Soc. Chim. Belges, 57, 252 (1948).
4. A. Katchalsky and J. Gills, Rec. Trav. Chim., 68, 879 (1949).

5. A. Arnold and J. Th. G. Overbeek, Rec. Trav. Chim., 69, 192 (1950).

6. F. E. Harris and S. A. Rice, J. Phys. Chem., 58, 725, 733 (1954).

7. G. S. Hartley and J. W. Roe, Trans. Faraday Soc., 36, 101 (1940).

8. M. Nagasawa, T. Murase, and K. Kondo, J. Phys. Chem., 69, 4005 (1965).

9. G. Körtun, W. Vogel, and K. Andrussov, Ed., "Dissociation Constants of Organic Acids in Aqueous Solution," Butterworths, London, 1961.

10. J. C. Leyte and M. Mandel, J. Polym. Sci., A, 2, 1879 (1964).

11. Y. Kawaguchi and M. Nagasawa, J. Phys. Chem., 73, 4382 (1969).

12. Y. Muroga, I. Noda and M. Nagasawa, J. Phys. Chem., 73, 667 (1969).

13. M. Nagasawa and A. Holtzer, J. Am. Chem. Soc., 86, 531 (1964).

14. Y. Sakaguchi, J. Nishino, K. Tamaki, and T. Ueda, Kobunshi Kagaku, 25, 273 (1968).

15. Y. Sakaguchi, J. Nishino, and K. Tamaki, Kobunshi Kagaku, 28, 789 (1971).

16. M. L. Miller and J. Skogman, J. Polym. Sci., A, 2, 4551 (1964). 